\title{
Editorial
}
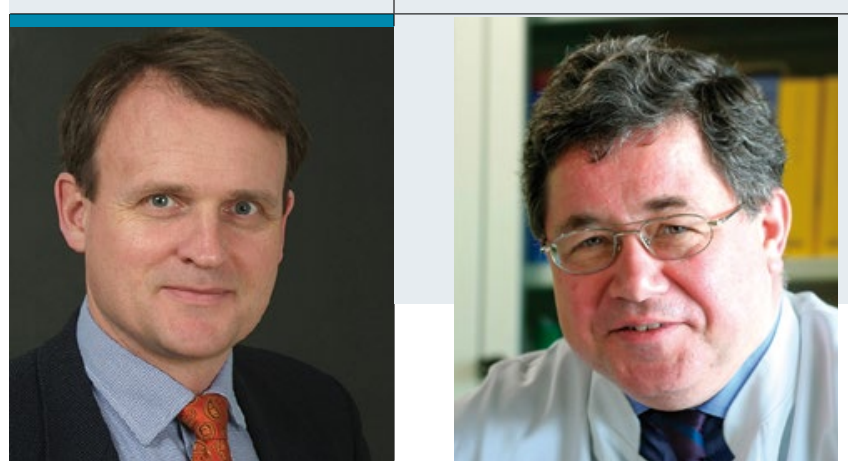

"Die neu gegründete Allergieliga soll

eine umfassende Verbesserung der

Versorgung von Patienten mit Allergien

in Deutschland bewirken."

\section{AeDA und DGAKI im Schulterschluss}

Prof. Dr. Thilo Jakob, Klinik für Dermatologie und Allergologie, Universitätsklinikum Gießen und Marburg, Standort Gießen

Prof. Dr. Hans F. Merk, Hautklinik der Medizinischen Fakultät, Universitätsklinikum der RWTH Aachen

$\mathrm{D}$ er 11. Deutsche Allergiekongress in Berlin hat mit hochkarätigen Beiträgen überzeugt und war mit über 1.300 Teilnehmern rekordverdächtig. Für unsere Leser, denen es nicht möglich war, den Kongress zu besuchen, haben wir die wesentlichen Highlights in der Rubrik „Allergologie aktuell“ zusammengefasst (S. 50). Momentaufnahmen, die während des Kongresses entstanden sind, finden Sie auf den Verbandsseiten (siehe S. 60 ff.). Besonderen Dank gilt den Organisatoren Frau Prof. Dr. Susanne Lau und Herrn PD Dr. Jörg KleineTebbe, die ein wunderbar abwechslungsreiches Programm finalisiert und mit viel Beharrlichkeit die Referenten im Vorfeld darauf eingeschworen haben, praxisrelevante Aspekte in ihren Beiträgen in den Vordergrund zu stellen. Insbesondere die Vorgabe, dass ein Drittel der Vortragszeit für Fragen vorbehalten ist, hat den nötigen Raum geschaffen, um ausführlich zu diskutieren. Hiervon wurde ausgiebig Gebrauch gemacht. Wir sollten diese Regelung daher für die künftigen Kongresse beibehalten.

Neben den wissenschaftlichen und klinischen Neuigkeiten gab es auf dem 11. Deutschen Allergiekongress auch eine Neuentwicklung im Bereich unserer Verbandsstrukturen. Am 28. September 2016 wurde die Deutsche Allergieliga e.V. gegründet. Hierbei handelt es sich um einen Schulterschluss der beiden deutschen interdisziplinären Allergiefachgesellschaften AeDA und DGAKI, mit der langfristigen Zielsetzung, eine umfassende Verbesserung der Versorgung von Patienten mit Allergien in Deutschland zu bewirken. Diese Ziele sollen erreicht werden durch entsprechende Öffentlichkeitsarbeit wie Aufklärung der Bevölkerung über Bedeutung, Verbreitung, Ursachen, Vorbeugen und Behandlungsmöglichkeiten von Allergien sowie durch die Erarbeitung von Empfehlungen und Positionspapieren zur Vorbeugung, Früherkennung, Diagnostik, Therapie, Nachsorge und Rehabilitation in Zusammenarbeit mit allen relevanten Interessensgruppen. Pate für das Konzept stand die 1979 gegründete Atemwegsliga, die sich sehr erfolgreich für eine optimale Versorgung von Patienten mit Atem- wegserkrankungen einsetzt. Nach abschließenden juristischen Prüfungen wird die Allergieliga ihre Arbeit zu Beginn 2017 aufnehmen. Bisher sind die DGAKI und der AeDA vertreten, weitere Fachgesellschaften, die sich um die Versorgung allergiekranker Patienten kümmern, sind herzlich eingeladen, sich der Allergieliga anzuschließen.

Unerfreuliche Nachrichten hören wir von der Stiftung Deutscher Polleninformationsdienst (PID), die auf die prekäre Situation der Pollenzählungen in Deutschland aufmerksam macht. In Zeiten von wirtschaftlicher Effizienz und hohem Kostendruck scheint die Pollenzählung in Deutschland in Gefahr $\mathrm{zu}$ sein. Anders als in anderen europäischen Ländern gab es bisher keine staatliche Unterstützung für die Arbeit des PID. Die bisherige Finanzierung erfolgte durch den begrenzten Verkauf der Daten für Studienzwecke. Diese finanziellen Ressourcen sind aktuell erschöpft, sodass mit Ablauf des Jahres 2016 der Betrieb der PID-Pollenmessstationen ohne zusätzliche Mittel nicht aufrechterhalten werden kann. In einem aktuellen Appell wendet sich nun der PID an das Bundesministerium für Gesundheit, um die Arbeit der Pollenzählungen finanziell zu unterstützen. Wir würden uns freuen, wenn Sie diesen Appell unterstützen. Weitere Details und ein entsprechendes Unterschriftenblatt finden Sie auf S. 71/72.

In der aktuellen Ausgabe bieten wir Ihnen zudem einen CME-Beitrag über verschiedene Spielvarianten ekzematöser Hauterkrankungen von Prof. Dr. Bettina Wedi, Hannover, eine Übersichtsarbeit zum komplexen Thema der Schalentier- und Krustazeen-Allergie von Prof. Dr. Andreas Lopata, Townsville, Australien, und eine Originalarbeit zur aktuellen Prävalenz allergischer Erkrankungen bei Schülern im Alter von 9 Jahren, die erneut das große Krankheits- und Belastungspotential dieser Erkrankungen im Schulalter belegt.

Wir wünschen Ihnen eine genussvolle Lektüre der aktuellen Ausgabe an den nun vor uns liegenden langen Herbstabenden.

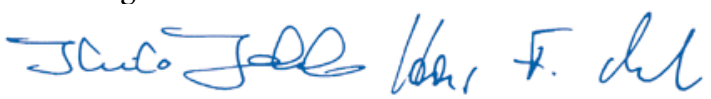

